\title{
Cross-Sectional and Longitudinal Construct Validity of the Generic KINDL-A(dult)B(rief) Questionnaire in Adults with Thrombophilia or with Hereditary and Acquired Bleeding Disorders
}

\author{
Bruno Neuner $^{a}$ Sylvia von Mackensen ${ }^{b}$ Bettina Kiesau ${ }^{c}$ Henning Krampe $^{a}$ \\ William J. McCarthy ${ }^{d}$ Sarah Reinkec, e Dorothee Kowalskic Maria Shneyder ${ }^{c}$ \\ Hartmut Clausnizer ${ }^{c}$ Angela Rockec ${ }^{c}$ Ralf Junker ${ }^{c}$ Ulrike Nowak-Göttlc, e \\ a Department of Anesthesiology and Intensive Care Medicine, Charité - Universitätsmedizin Berlin, Campus Charité

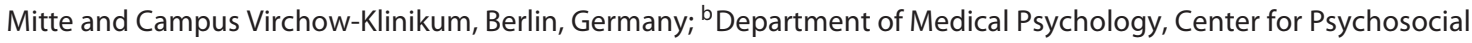 \\ Medicine, University Medical Centre Hamburg-Eppendorf, Hamburg, Germany; ${ }^{C}$ Institute for Clinical Chemistry \\ and Coagulation Center, University Hospital Schleswig Holstein, Lübeck/Kiel, Germany; ${ }^{\text {d}}$ Fielding School of Public \\ Health and Jonsson Comprehensive Cancer Center, Center for Cancer Prevention and Control Research, University \\ of California Los Angeles (UCLA), Los Angeles, CA, USA; ${ }^{e}$ Department of Pediatric Oncology and Hematology, \\ University Childrens' Hospital, Münster, Germany
}

\section{Keywords}

Health-related quality of life · Hereditary and acquired bleeding disorder - Longitudinal construct validity

\begin{abstract}
Background/Aims: The newly adapted generic KINDL$\mathrm{A}$ (dult)B(rief) questionnaire showed satisfactory cross-sectional psychometric properties in adults with bleeding disorders or thrombophilia. This investigation aimed to evaluate its cross-sectional and longitudinal construct validity. Methods: After ethical committee approval and written informed consent, 335 patients (mean age $51.8 \pm 16.6$ years, $60 \%$ women) with either predominant thrombophilia ( $n=$ $260)$ or predominant bleeding disorders $(n=75)$ participated. At baseline, patients answered the KINDL-AB, the MOS 36-item Short-Form Health Survey (SF-36), and the EQ-5D$3 \mathrm{~L}$. A subgroup of 117 patients repeated the questionnaire after a median follow-up of 2.6 years (range: $0.4-3.5$ ). A priori hypotheses were evaluated regarding convergent correla-
\end{abstract}

tions between KINDL-AB overall well-being and specific subscales, EQ-5D-3L index values (EQ-IV), EQ-5D visual analog scale (EQ-VAS), and SF-36 subscales. Results: Contrary to hypothesis, baseline correlations between the KINDL-AB and EQ-IV/EQ-VAS were all moderate while, as hypothesized, several KINDL-AB subscales and SF-36 subscales correlated strongly. At follow-up, no significant changes in all three instruments occurred. Correlations between instruments over the follow-up were mostly moderate and partially strong. Contrary to hypothesis but consistent with no significant changes in health-related quality of life, convergent correlations between changes in KINDL-AB overall well-being, physical and psychological well-being, and EQ-IV/EQ-VAS were all weak. Conclusions: While repeated measures of KINDL-AB showed moderate to strong correlations, changes in KINDL-AB overall well-being and subscales correlated more weakly than expected with changes involving two established instruments of generic health status.

Cㄱ 2020 S. Karger AG, Base

karger@karger.com
www.karger.com/aha
Karger $V^{\prime /}$




\section{Introduction}

There are several questionnaires evaluating diseasespecific quality of life in children, adolescents, and adults with thrombophilia disorders including thromboembolism and pediatric stroke $[1,2]$ or with hereditary and acquired bleeding disorders (HABD) [3-9]. In patients with bleeding disorders, health-related quality of life (HRQoL) varies with occurrence of bleeds, pain, and related limitations in everyday activities [10]. Furthermore, disease-specific questionnaires do not easily allow comparisons between population-based health measures or population-wide health assessments, and they can impede the conduct of comparative cost-benefit and costeffectiveness analyses [11]. In such situations or when comparisons with patients with other disorders are warranted [12], generic HRQoL measures may add useful information to or partially replace disease-specific health measures. But generic health status measures also have limitations when applied to longitudinal research involving different age groups, especially when studying longitudinal changes of HRQoL through childhood and adolescence into adulthood. This led to the idea of modifying an established HRQoL questionnaire, the generic KINDL$\mathrm{R}$ questionnaire [13, 14] (www.kindl.org), originally meant for use from early childhood up to adolescence ( $\leq 16$ years), but not beyond this age limit. In order to allow its continual use into the age groups $>16$ years, the KINDL-A(dult)B(rief) questionnaire with a reduced overall number of items and a reduced number of subscales was adapted and cross-sectionally validated in a group of adult patients with thrombophilia/HABD as well as in healthy adult blood donors [15]. For the period being evaluated, goodness-of-fit indices were promising and the factor loadings, especially in the study population of patients, were reasonable [15]. Before applying the newly adapted questionnaire to children and adolescents in transition to adulthood, we intended first to study its cross-sectional and longitudinal construct validity in a group of adult patients with thrombophilia or HABD.

\section{Materials and Methods}

\section{Inclusion Criteria}

Adult patients with thrombophilia or HABD in routine treatment in the outpatient department of the Institute for Clinical Chemistry and Coagulation Center of the University Hospital Schleswig Holstein, Campus Kiel. Patients were usually referred by their family doctors or by peripheral hospitals to diagnose the underlying causes of clinically relevant thromboses or bleeding diathesis caused via inherited or acquired coagulation factor deficiencies. Since February
2014, patients have been continuously enrolled up to the present day. Patients enrolled from February 2014 to February 2016 were already included in the cross-sectional study reporting the creation and initial validation of the KINDL-AB questionnaire [15].

\section{Exclusion Criteria}

Patients aged less than 18 years, non-German speaking patients, patients unable to understand and answer the questionnaires, or patients without written informed consent.

\section{Study Population}

During one of their routine clinical visits, potentially eligible patients were invited to participate in the study and, after written informed consent, completed the baseline questionnaire that included questions about basic socioeconomic characteristics and generic HRQoL scales. The follow-up questionnaire was administered again during one of the patients' follow-up visits or sent to the patients' homes.

\section{Measures}

The KINDL-AB has 12 items in five subscales and is a short form of the 24-item KINDL-A(dult) questionnaire, an adaptation of the KINDL-R [15]. Altogether, 5 items of the KINDL-R were modified ( 2 items in the family-related well-being subscale and 3 in the school-related well-being subscale). Application of this 24item KINDL-A questionnaire to a subgroup of the analyzed patients revealed several items with insufficiently large factor loadings (below 0.7 ) on their latent variables. This led to deletion of scale items and to the deletion of the self-esteem subscale. The only item with acceptable factor loading in the self-esteem subscale was collapsed with 2 items of emotional well-being to form a newly defined psychological well-being subscale. The newly adapted KINDL-AB showed factor loadings above 0.6 for all 12 items and goodness-of-fit indices (GFI) of moderately acceptable fit (root mean square error of approximation, RMSEA <0.08) and more highly acceptable model fit $(\mathrm{GFI}>0.9)$, respectively. However, the adjusted GFI did not meet the 0.9 threshold (AGFI $=0.86)$ and the Bentler comparative fit index (CFI) fell just short of meeting the 0.95 threshold (Bentler CFI $=0.94$ ) [16]. In a group of healthy blood donors, factor loadings were weaker but GFI were more promising (RMSEA $<0.05$, indicating good fit [16]; GFI, AGFI, Bentler CFI, all $>0.95$, indicating acceptable fit).

The EQ-5D-3L [17] with its two summary measures, the EQ$5 \mathrm{D}-3 \mathrm{~L}$ index value (EQ-IV) and the EQ-5D visual analog scale (EQ-VAS), served together with the MOS 36-item Short-Form Health Survey (SF-36) $[18,19]$ as the gold standard for measures in HRQoL and were administered both at baseline and at followup. The EQ-5D-3L is composed of five domains (mobility, selfcare, usual activities, pain/discomfort, and anxiety/depression) concerning one's current health status. EQ-5D-5L health states can be converted into a single index value (EQ-IV). Additionally, one's current subjective health state was measured with a single measure on a "thermometer" scaled from 0 to 100 (EQ-VAS).

\section{Cross-Sectional and Longitudinal Construct Validity}

A priori hypotheses were formulated and translated into hypotheses concerning the expected magnitude of specific correlations. According to Evans [20], "very weak" correlations range from 0.00 to 0.19 , "weak" correlations range from 0.20 to 0.39 , "moderate" correlations range from 0.40 to 0.59 , "strong" correla- 
Table 1. Sociodemographic patient characteristics at baseline, $n=335$

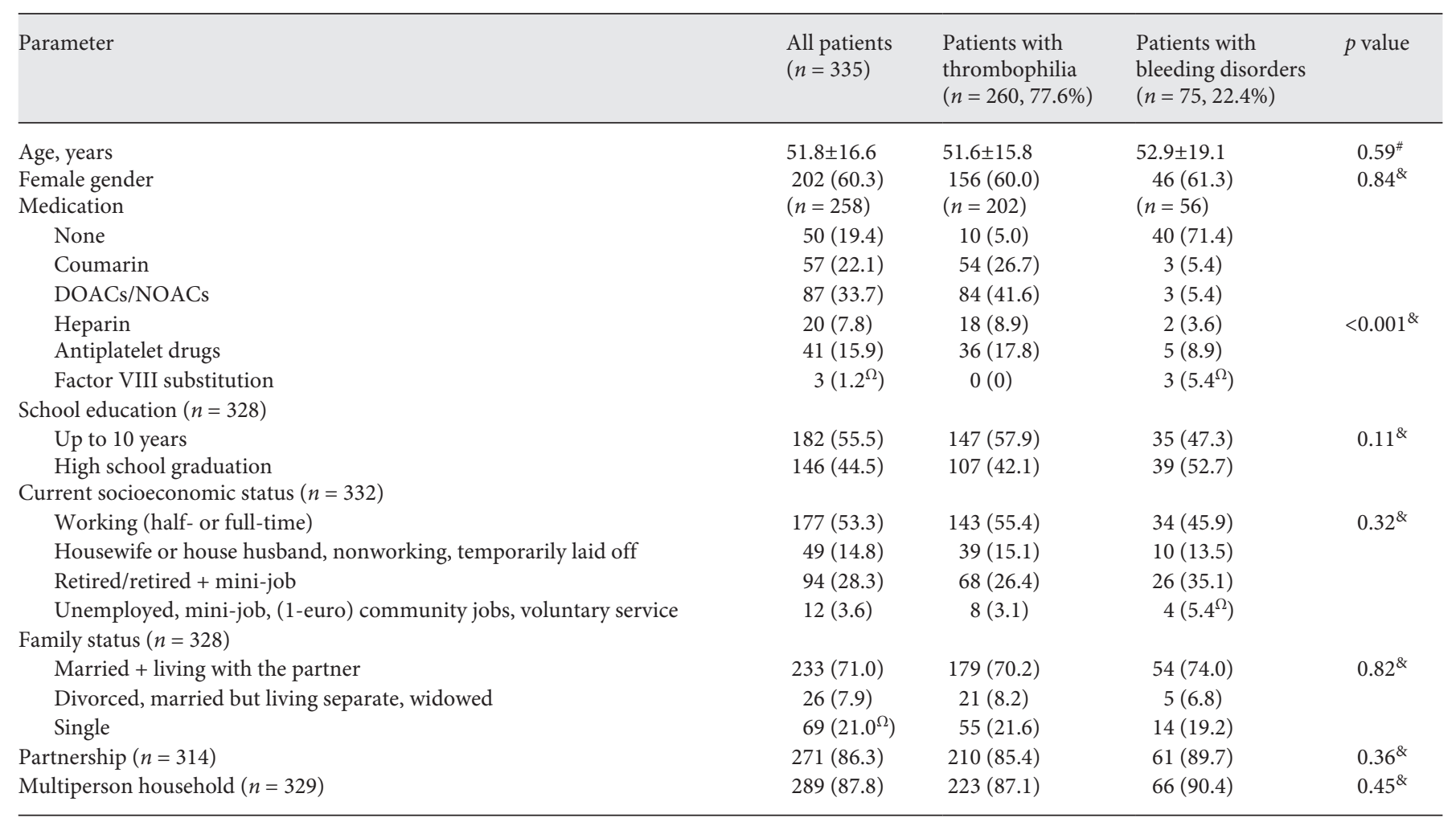

Data are presented as $n(\%)$ or mean $\pm \mathrm{SD}$, as appropriate. SD, standard deviation; DOAC, directly acting oral anticoagulants; NOAC, novel oral anticoagulants. ${ }^{\Omega}$ Does not sum up to $100 \%$ because of rounding errors; ${ }^{*}$ Student's $t$ test; ${ }^{\&} \chi^{2}$ test.

tions range from 0.60 to 0.79 , and "very strong" correlations range from 0.80 to 1.00 . In particular, the following hypotheses involving correlations between KINDL-AB overall well-being, KINDL-AB subscales, and gold standard measures of generic health status were evaluated:

- Strong cross-sectional correlations and at least moderate longitudinal correlations between KINDL-AB overall well-being and EQ-IV, EQ-VAS, SF-36 general health perception, and SF36 vitality.

- Strong cross-sectional correlations and at least moderate longitudinal correlations between KINDL-AB physical well-being and EQ-IV, EQ-VAS, SF-36 physical functioning, SF-36 physical role functioning, and SF-36 vitality.

- Strong cross-sectional correlations and at least moderate longitudinal correlations between KINDL-AB psychological wellbeing and EQ-IV, EQ-VAS, SF-36 vitality, SF-36 emotional role functioning, and SF-36 mental health.

- Strong cross-sectional correlations and at least moderate longitudinal correlations between KINDL-AB family-related wellbeing and SF-36 social role functioning.

- Strong cross-sectional correlations and at least moderate longitudinal correlations between KINDL-AB friend-related wellbeing and SF-36 social role functioning.

- Strong cross-sectional correlations and at least moderate longitudinal correlations between KINDL-AB education/workrelated well-being and SF-36 social role functioning.
Correlation analyses can be easily biased by outliers [21] and non-normally distributed data [22]. The assumptions for applying parametric correlation analyses such as nonexistent extreme outliers (for this study defined as values more than 3 times the interquartile range distant from the median) were tested by boxplots and the existence of a normal distribution was tested by evaluating the skewness (normal distribution defined as skewness between -1 and +1 ). Since several continuous variables included extreme outliers and some continuous variables featured non-normally distributed data (for details, see online suppl. Table 1; for all online suppl. material, see www.karger.com/doi/10.1159/000507602), all correlation analyses, after removal of extreme outliers, were conducted using the nonparametric Spearman's rho $(\rho)$ correlation measure.

\section{Descriptive Statistics}

Patients' basic characteristics, when values were normally distributed, are displayed as means accompanied by standard deviations in parentheses, while variables with skewed values are displayed as median values (range). Count data are displayed as absolute and relative frequencies. Differences between two independent groups (Tables 1,2 ) were evaluated using the $\chi^{2}$ test in analyses involving binary and ordinal variables. Student's $t$ test and the Mann-Whitney $U$ test were used in analyses involving continuous, normally distributed and continuous, non-normally distributed variables, respectively. Changes in HRQoL at follow- 
Table 2. Health-related quality of life measures at baseline, $n=335$

\begin{tabular}{|c|c|c|c|c|}
\hline \multicolumn{5}{|l|}{ KINDL-AB } \\
\hline Overall well-being & $67.2 \pm 14.7$ & $67.3 \pm 15.2$ & $66.6 \pm 12.9$ & $0.71^{\#}$ \\
\hline Physical well-being & $53.4 \pm 22.9$ & $54.5 \pm 23.0$ & $49.9 \pm 22.2$ & $0.13^{\#, \&}$ \\
\hline Friend-related well-being $(n=321)$ & $69.7 \pm 18.2$ & $68.9 \pm 19.1$ & $72.2 \pm 14.6$ & $0.12^{\#, \&}$ \\
\hline Education/work-related well-being $(n=227)$ & $74.9 \pm 19.9$ & $75.5 \pm 18.8$ & $72.2 \pm 23.6)$ & $0.31^{\#, \&}$ \\
\hline EQ-IV $(n=242)$ & $0.80(-0.18$ to 1.00$)$ & $0.80(-0.07$ to 1.00$)$ & $0.76(-0.18$ to 1.00$)$ & $0.090^{\$}$ \\
\hline EQ-VAS $(n=249)$ & $75(0$ to 100$)$ & $80(0$ to 100$)$ & $75(35$ to 100$)$ & $0.071^{\$}$ \\
\hline \multicolumn{5}{|l|}{ SF-36 } \\
\hline Physical functioning $(n=136)$ & $76.9 \pm 22.5$ & $76.4 \pm 22.3$ & $78.7 \pm 24.0$ & $0.64^{\#, \S}$ \\
\hline Social role functioning $(n=134)$ & $76.1 \pm 26.9$ & $75.6 \pm 28.0$ & $78.2 \pm 22.6$ & $0.65^{\#, \S}$ \\
\hline Emotional role functioning $(n=132)$ & $77.1 \pm 26.1$ & $77.1 \pm 25.1$ & $77.2 \pm 30.2$ & $0.99^{\#, \S}$ \\
\hline Mental health & $70.6 \pm 17.9$ & $71.0 \pm 18.5$ & $68.9 \pm 15.6$ & $0.59^{\#, \S}$ \\
\hline
\end{tabular}

Data are presented as mean \pm SD or median (range), as appropriate. SD, standard deviation; KINDL-AB, adult brief version of the revised KINDL questionnaire; EQ-IV, EQ-5D-3L index value of the EQ-5D-3L questionnaire; EQ-VAS, EQ-5D visual analog scale; SF36, Short-Form (36-Item) Health Survey. ${ }^{\#}$ Student's $t$ test; ${ }^{\$}$ Mann-Whitney U test; ${ }^{\&}$ significance level Bonferroni adjusted to $p<0.01$ (five subscales); ${ }^{\S}$ significance level Bonferroni adjusted to $p<0.006$ (eight subscales).

Table 3. Health-related quality of life, measured with the KINDL-AB, in patients with complete baseline and follow-up assessments, $n=60-117$

\begin{tabular}{|c|c|c|c|c|c|}
\hline KINDL-AB, overall well-being and subscales & $\begin{array}{l}\text { Baseline } \\
(\text { mean } \pm \mathrm{SD})\end{array}$ & \multicolumn{4}{|l|}{ Follow-up } \\
\hline Overall well-being $(n=109)$ & $69.8 \pm 13.9$ & $71.2 \pm 14.2$ & $1.4(-0.7$ to 3.5$)$ & 0.20 & $0.644^{* *}$ \\
\hline Physical well-being $(n=113)$ & $57.0 \pm 23.2$ & $60.9 \pm 22.6$ & $3.9(-0.2$ to 8.1$)$ & 0.06 & $0.493^{* *}$ \\
\hline Psychological well-being $(n=117)$ & $68.5 \pm 19.1$ & $71.1 \pm 18.1$ & $2.6(-0.6$ to 5.9$)$ & 0.11 & $0.504^{* *}$ \\
\hline Education/work-related well-being $(n=60)$ & $78.8 \pm 20.8$ & $74.6 \pm 24.6$ & $-4.2(-9.8$ to 1.5$)$ & 0.14 & $0.530^{* *}$ \\
\hline
\end{tabular}

KINDL-AB, adult brief version of the revised KINDL questionnaire; SD, standard deviation; CI, confidence interval. ${ }^{*} *<<$ $0.001 .^{\mathrm{a}}$ Dependent $t$ test for paired samples; the significance level of the $p$ value of the five subscales was Bonferroni adjusted to $p<0.01$. b According to Evans[11], correlations in the range of 0.00-0.19 are "very weak," $0.20-0.39$ are "weak," $0.40-0.59$ are "moderate," 0.60 0.79 are "strong," and $0.80-1.0$ are "very strong".

up (Tables 3, 4) were evaluated using Student's $t$ test for paired samples in continuous, normally distributed variables and Wilcoxon signed-rank test in continuous, non-normally distributed variables. $p$ values for analyses in the five subscales of the KINDL$\mathrm{AB}$ were Bonferroni-corrected to a significance level of $p<0.01$
(Tables 2, 3) and to $p<0.00625$ in the eight subscales of the SF-36 (Tables 2, 4). All statistical analyses were performed using IBM SPSS Statistics for Windows (Version 23.0, released 2015; IBM Corp., Armonk, NY, USA). 
Table 4. Health-related quality of life measured with the EQ-5D-3L and the SF-36, in patients with complete baseline and follow-up assessments, $n=36-96$

\begin{tabular}{|c|c|c|c|c|c|}
\hline & Baseline & Follow-up & $\begin{array}{l}\text { Mean change } \\
(95 \% \mathrm{CI})\end{array}$ & $p$ value & $\begin{array}{l}\text { Spearman } \\
\text { correlation }^{2}\end{array}$ \\
\hline EQ-IV $(n=90)$ & $0.80(-0.18$ to 1.00$)$ & $0.80(-0.18$ to 1.00$)$ & - & $0.80^{\S}$ & $0.597^{* *}$ \\
\hline \multicolumn{6}{|l|}{ SF-36 $(n=36-38)$} \\
\hline Physical functioning & $80.4 \pm 19.2$ & $83.2 \pm 19.4$ & $2.8(-2.8$ to 8.4$)$ & $0.33^{\$, \Omega}$ & $0.569^{* *}$ \\
\hline General health perceptions & $65.2 \pm 20.2$ & $64.5 \pm 18.7$ & $-0.7(-6.7$ to 5.2$)$ & $0.80^{\$, \Omega}$ & $0.621^{* *}$ \\
\hline Vitality & $60.4 \pm 17.4$ & $60.2 \pm 18.3$ & $-0.2(-4.8$ to 4.5$)$ & $0.94^{\$, \Omega}$ & $0.583^{* *}$ \\
\hline Social role functioning & $77.4 \pm 26.4$ & $78.1 \pm 27.3$ & $0.7(-6.2$ to 7.5$)$ & $0.84^{\$, \Omega}$ & $0.611^{* *}$ \\
\hline Emotional role functioning & $76.8 \pm 23.2$ & $79.5 \pm 23.6$ & $2.7(-4.8$ to 10.2$)$ & $0.47^{\$, \Omega}$ & $0.472^{*}$ \\
\hline Mental health & $72.9 \pm 17.1$ & $72.8 \pm 17.5$ & $-0.1(-4.5$ to 4.2$)$ & $0.95^{\$, \Omega}$ & $0.670^{* *}$ \\
\hline
\end{tabular}

Data are presented as mean \pm SD or median (range), as appropriate. EQ-IV, EQ-5D-3L index value of the EQ-5D-3L questionnaire; EQ-VAS, EQ-5D visual analog scale; SF-36, Short Form (36-Item) Health Survey: SD, standard deviation; CI, confidence interval. * $p<$ 0.05 ; $^{* *} p<0.001 .{ }^{\S} \mathrm{Wilcoxon}$ signed-rank test; ${ }^{\$}$ dependent $t$ test for paired samples; ${ }^{\Omega}$ the $p$ value of the eight subscales of the SF-36 was Bonferroni adjusted to $p<0.006$. "According to Evans [11], correlations in the range of 0.00-0.19 are "very weak," $0.20-0.39$ are "weak," 0.40-0.59 are "moderate," $0.60-0.79$ are "strong," and 0.80-1.0 are "very strong".

\section{Results}

Overall, 335 patients participated. Patients with predominant thrombotic events composed the majority of patients (78\%). As shown in online supplementary Figure 1 , the majority of patients had peripheral thromboses only $(n=96)$, followed by pulmonary embolisms $(n=82)$ either in combination with peripheral thromboses or as single diagnoses, or cerebral thromboses $(n=43)$. Online supplementary Figure 2 depicts the diagnoses of the 75 patients with bleeding disorders, mostly von Willebrand disease and hemophilia $(n=28)$, secondary drug-induced coagulation factor deficiencies $(n=24)$, or rare inherited coagulation factor deficiencies $(n=23)$. The mean (standard deviation) age of all patients was 51.8 (16.6) years with an age range from 18 to 89 years. The majority of patients were women (60.3\%). As shown in Table 1, there were no differences in sociodemographic data at baseline between both patient groups; no significant differences were found also for the different generic HRQoL measures (see Table 2). Patient groups differed in their medication at the time of the study (the information on medication was available from 258 patients, i.e., $77 \%$ of all study participants). Most patients with bleeding disorders $(71 \%)$ were without coagulation medication, while $95 \%$ of patients with thrombophilia took at least one or a combination of inhibitory medications. The different classes of medication are listed in Table 1.
All 335 patients answered the KINDL-AB questionnaire; 249 patients additionally answered the EQ-5D-3L and 136 the SF-36. In the EQ-IV and EQ-VAS, there was a tendency for patients with bleeding disorders to show lower values than patients with thrombophilia $(p=0.090$ and $p=0.071$ ). Through the end of April 2019 and after a median follow-up of 2.6 years (range: $0.4-3.5$ ), complete pairs of baseline/follow-up assessments existed in 117 study participants who completed the KINDL-AB (see Table 3). There was a nonsignificant increase in overall well-being: +1.4 (95\% confidence interval -0.7 to 3.5 ), $p=0.20$. The KINDL-AB overall well-being scores at baseline and follow-up were strongly correlated. No significant changes in subscales were observed at follow-up and correlations between baseline and follow-up scores were moderate (except for KINDL-AB friend-related well-being scores, which showed a strong correlation: Spearman's $\rho=0.658, p<0.001$ ).

Overall, 96 study participants had complete pairs of baseline/follow-up EQ-5D-3L assessments and 38 study participants had complete pairs of baseline/follow-up SF36 assessments (see Table 4). Changes in EQ-IV from baseline to follow-up were not significant but significant changes in EQ-VAS scores occurred at follow-up ( $p=$ 0.002 ). Correlations between baseline and follow-up EQIV and EQ-VAS scores were in the upper moderate range. Correlations between baseline and follow-up SF-36 subscale scores were strong for three out of eight subscales: 
Table 5. Cross-sectional convergent correlations between different measures of health-related quality of life: Spearman correlation coefficients $\rho$ at baseline, $n=92-248$

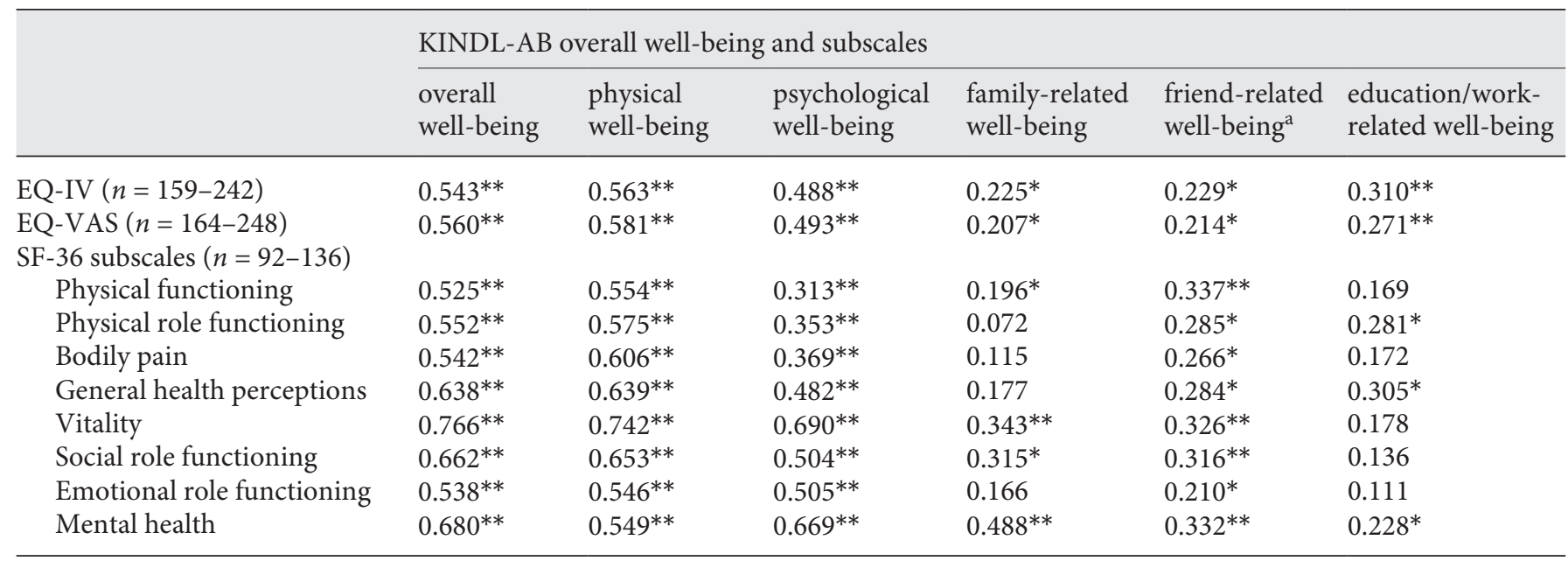

KINDL-AB, adult brief version of the revised KINDL questionnaire; EQ-IV, EQ-5D-3L index value of the EQ-5D-3L questionnaire; EQ-VAS, EQ-5D visual analog scale; SF-36, Short Form (36-Item) Health Survey. ${ }^{*} p<0.05$; ${ }^{* *} p<0.001$. According to Evans [11] correlations in the range of 0.00-0.19 are "very weak," $0.20-0.39$ are "weak," $0.40-0.59$ are "moderate," $0.60-0.79$ are "strong," and 0.80-1.0 are "very strong". a 5 extreme outliers removed.

mental health, social role functioning, and general health perception. The bodily pain subscale showed only weak correlations. Physical functioning as well as vitality showed correlations in the upper moderate range.

Convergent correlations were mostly weaker than hypothesized (see Table 5). For example, cross-sectional convergent correlations between KINDL-AB overall well-being and physical well-being and EQ-5D-3L measures all exceeded the 0.5 correlation threshold, but not the 0.6 threshold for "strong" correlations. KINDL-AB psychological well-being was correlated with EQ-5D-3L measures only close to the 0.5 threshold. KINDL-AB physical wellbeing did, as hypothesized, strongly correlate with vitality but slightly failed to meet the "strong" correlation threshold with SF-36 physical functioning and SF-36 physical role functioning. Furthermore, the KINDL-AB overall well-being showed - contrary to hypothesis - near-to-strong or strong correlations with all other SF-36 subscales as well. KINDL-AB psychological well-being, did, as hypothesized, strongly correlate with two of three SF-36 subscales (mental health and vitality). Contrary to hypothesis, the remaining three KINDL-AB scales (family-related well-being, friendrelated well-being, and education/work-related well-being) did not even moderately correlate with the SF-36 social role functioning subscale. Regarding longitudinal construct validity (see Table 6) and contrary to hypothesis, changes in KINDL-AB overall well-being, physical well-being, and psychological well-being and in EQ-5D-3L measures were only weakly correlated but consistent with there being no significant change in HRQoL, no matter how assessed. Changes in KINDL-AB physical well-being moderately correlated with changes in SF-36 physical role functioning and vitality, but not with changes in physical functioning. Changes in KINDL-AB psychological well-being moderately correlated with changes in SF-36 mental health, but not with changes in SF-36 emotional role functioning and vitality. The remaining three KINDL-AB scales (family-related well-being, friend-related well-being, and education/ work-related well-being), contrary to hypothesis, did not correlate even moderately with the SF-36 social role functioning subscale.

\section{Discussion}

In a study population of adult patients with thrombophilia or with hereditary and acquired bleeding disorders, the 12 -item version for adults of a modified version of the KINDL-R questionnaire, the KINDL-AB, showed no statistically significant change over a median follow-up of 2.4 years. HRQoL at follow-up was strongly correlated with HRQoL at baseline. At baseline, KINDL-AB overall well-being, physical well-being, and psychological wellbeing correlated moderately with EQ-5D-3L measures 
Table 6. Longitudinal convergent correlations between different measures of health-related quality of life: Spearman correlation coefficients $\rho$ of changes in quality of life from baseline to follow-up, $n=20-96$

\begin{tabular}{|c|c|c|c|c|c|c|}
\hline $\begin{array}{l}\text { Changes in EQ-5D-3L and } \\
\text { SF-36 from baseline to follow-up }\end{array}$ & \multicolumn{6}{|c|}{ Changes in KINDL-AB overall well-being and subscales from baseline to follow-up } \\
\hline EQ-IV ${ }^{\mathrm{c}}(n=42-89)$ & $0.256^{*}$ & $0.319^{*}$ & 0.210 & -0.009 & -0.060 & 0.085 \\
\hline $\mathrm{EQ}-\mathrm{VAS}^{\mathrm{a}}(n=44-96)$ & $0.282^{*}$ & $0.295^{*}$ & $0.272^{*}$ & 0.054 & 0.013 & 0.139 \\
\hline \multicolumn{7}{|l|}{ SF-36 subscales $(n=20-39)$} \\
\hline Bodily pain & 0.074 & 0.230 & 0.080 & -0.157 & 0.154 & -0.014 \\
\hline General health perceptions & 0.194 & $0.328^{*}$ & 0.203 & 0.202 & 0.093 & -0.285 \\
\hline Vitality & 0.189 & $0.436^{*}$ & 0.278 & 0.015 & -0.117 & -0.145 \\
\hline Social role functioning & 0.332 & 0.297 & $0.413^{*}$ & 0.274 & $0.344^{*}$ & -0.222 \\
\hline Emotional role functioning & 0.266 & $0.412^{*}$ & 0.188 & 0.196 & 0.072 & -0.138 \\
\hline
\end{tabular}

KINDL-AB, adult brief version of the revised KINDL questionnaire; EQ-IV, EQ-5D-3L index value of the EQ-5D-3L questionnaire; EQ-VAS, EQ-5D visual analog scale; SF-36, Short Form (36-Item) Health Survey. ${ }^{*} p<0.05$; ${ }^{* *} p<0.001$. According to Evans [11], correlations in the range of 0.00-0.19 are "very weak," $0.20-0.39$ are "weak," $0.40-0.59$ are "moderate," $0.60-0.79$ are "strong," and 0.80-1.0 are "very strong". a 1 outlier removed. ${ }^{\text {b }} 2$ outliers removed. ${ }^{c} 3$ outliers removed.

and strongly with single SF-36 subscales. Correlations of changes over time were generally weak.

The KINDL-AB questionnaire is meant to allow a continual assessment of generic HRQoL in patients with thrombophilia or bleeding disorders from childhood throughout adulthood, when they were initially addressed with the KINDL-R $[13,14]$. The KINDL-R is only validated for use in children up to the age of 16 years. For assessing HRQoL in adult patients with thrombophilia or bleeding disorders, switching to an established HRQoL questionnaire such as the SF-36 or the EQ-5D was required prior to the development of the KINDL-AB questionnaire. So far, its basic psychometric properties, as presented here, seem promising. Its predominately moderate or weak cross-sectional and longitudinal convergent correlations with established HRQoL questionnaires seem to indicate that the KINDL-AB and established HRQoL questionnaires capture different aspects of HRQoL and thus overlap solely in selected domains.

Neither aspects of mobility (the first EQ-5D-3L dimension) nor aspects of self-care (the second EQ-5D-3L dimension) are measured with the KINDL-AB questionnaire. Pain and discomfort (the fourth EQ-5D-3L dimension) is no longer included in the physical well-being subscale of the KINDL-AB scale, as this item showed the lowest factor loading in this specific subscale (Table 4 in [15]) and was therefore deleted.
Factors other than nonoverlapping content may explain the low correlations: negative wording may add "variance to the item phrasing in addition to the variance for the item content" [23]. There is also evidence that "negatively worded items demonstrated longitudinal invariance" $[23,24]$. The focus on the illness rather than the wellness end of the HRQoL continuum in gold standard scales may likewise explain low cross-sectional or longitudinal correlations. Three of five EQ-5D-3L dimensions use the wording of "having problems," e.g., with walking about or with self-care, while especially the items of the last three subscales of the KINDL-AB, family-related well-being, friend-related well-being, and education/ work-related well-being use a positive wording ("got on well with," "felt fine," was a "success with," "got along well"). As mentioned earlier, aspects of family- or friendrelated well-being are not captured with the EQ-5D-3L, and therefore longitudinal convergent correlations close to zero, as observed here, could be expected (see Table 5, last three columns).

Stronger correlations, again both cross-sectionally as well as longitudinally, existed between the KINDL-AB questionnaire and specific SF-36 subscales. The physical well-being subscale of the KINDL-AB with its three items is composed of two negatively formulated items "felt ill" and "tired and worn-out," while the third item "strong and full of energy" is positively formulated. The wording 
of the three items of this specific KINDL-AB subscale is close to the wording of the four items of the vitality subscale of the SF-36 ("full of pep," "a lot of energy," "feel worn out" and "feel tired"). Therefore, the high convergent correlation at baseline and of changes over time in these two subscales of two different questionnaires may not be surprising. Physical role functioning (as well as emotional role functioning) of the SF-36 evaluates difficulties with "work" or "regular daily activities," which are not directly assessed with the three items of the KINDL$A B$ physical well-being subscale. Therefore, the observed Spearman correlations of $\rho=0.58$ at baseline (upperrange moderate correlation) and $\rho=0.42$ (moderate correlation) over time can be regarded nevertheless as promising.

The reduced number of responses, especially to the two items of KINDL-AB education and work-related well-being ("During the past week .... Doing the duties at school / in education / at the job was easy," KINDL-AB item 11, and "I found school / education / job interesting," KINDL-AB item 12) nevertheless indicate the need for revising these two items or their combination with an upstream question with a bipolar scale such as "Are you currently undergoing any formal education or full-time or part-time working?"). In the data presented here (see online suppl. Table 2, out-of-protocol analysis for this discussion), $93 \%$ of patients with half-time or full-time employment answered both KINDL-AB items 11 and 12, as did 56 and 57\%, respectively, of nonworking, temporary laid off participants and participants with mini-job (part-time) or "1-Euro" community job. Only 30 and $27 \%$ of the study participants who were retired (with or without additional mini-job) at the time of the interrogation answered these two questions, respectively. The KINDL-AB family-related well-being subscale was the subscale with the second greatest frequency of missing responses. Patients married and living with their partners at the time of the interrogation answered $100 \%$ of both items of this subscale (KINDL-AB item 13 "During the past four weeks, I got on well with my partner" and KINDL-AB item 14 "During the past four weeks, I felt fine at home"). In those who were divorced, married but living separately, or widowed, the percentages responding were 73 and $85 \%$, respectively, and in those who were currently single, the percentages responding were 67 and $75 \%$, respectively. Asking specifically about "going on well with a partner" may irritate respondents without a partner but does not necessarily explain the missing responses to the question on "feeling fine at home." Here, qualitative research is necessary to elucidate the underly- ing reluctance to answer specific items and to help revise specific items.

To summarize, the KINDL-AB overall well-being and its first two subscales, physical well-being and psychological well-being, show sufficient to good construct validity in both cross-sectional and longitudinal applications with regard to established generic HRQoL questionnaires. Its last three subscales add value with their assessment of specific domains of social well-being. Their mostly weak to sometimes very weak correlations with EQ-IV, EQ-VAS, as well as with the SF-36 indicate that they capture aspects of generic HRQoL not captured by these widely used and established questionnaires. However, the social domains of the KINDL-AB are not relevant to every patient. More selective application of the KINDL-AB according to medical condition or revision of these items need to be evaluated in further studies.

\section{Statement of Ethics}

The study was approved by the medical ethics committee of the University of Münster, Germany (2008-161-f-S) and the ethics committee of the University of Kiel (2016/9-B 304/16). All study participants provided their written informed consent.

\section{Disclosure Statement}

The authors declare that they have no conflicts of interest.

\section{Funding Sources}

This study was financed by unrestricted grants from Biotest AG, Langen, Germany, to Ulrike Nowak-Göttl.

\section{Author Contributions}

The study was planned and conducted by U.N.-G. who supervised B.K., S.R., D.K., M.S., H.C., and A.R., who screened potentially eligible patients, enrolled the study participants, and entered and managed the data. S.M. and H.K. supervised the psychometric analyses and provided important intellectual input. B.N. and U.N.-G., with assistance from W.J.C., wrote a first draft of the manuscript which was circulated among all authors. S.M. and R.J., together with the other authors, revised former versions of the manuscript, which was finalized by B.N. and W.J.C. All authors reviewed and approved the final version of this manuscript. 


\section{References}

1 Cohn DM, Nelis EA, Busweiler LA, Kaptein AA, Middeldorp S. Quality of life after pulmonary embolism: the development of the PEmb-QoL questionnaire. J Thromb Haemost. 2009 Jun;7(6):1044-6.

2 Fiume A, Deveber G, Jang SH, Fuller C, Viner $S$, Friefeld S. Development and validation of the Pediatric Stroke Quality of Life Measure. Dev Med Child Neurol. 2018 Jun;60(6):587-95.

3 Arranz P, Remor E, Quintana M, Villar A, Díaz JL, Moreno M, et al.; Hemofilia-QoL Group. Development of a new disease-specific quality-of-life questionnaire to adults living with haemophilia. Haemophilia. 2004 Jul; 10(4):376-82.

4 Limperg PF, Terwee CB, Young NL, Price VE, Gouw SC, Peters M, et al. Health-related quality of life questionnaires in individuals with haemophilia: a systematic review of their measurement properties. Haemophilia. 2017 Jul;23(4):497-510.

5 Rentz A, Flood E, Altisent C, Bullinger M, Klamroth R, Garrido RP, et al.; Members of the HAEMO-QoL-A Steering Committee. Cross-cultural development and psychometric evaluation of a patient-reported healthrelated quality of life questionnaire for adults with haemophilia. Haemophilia. 2008 Sep; 14(5):1023-34

6 Skinner MW, Chai-Adisaksopha C, Curtis R, Frick N, Nichol M, Noone D, et al. The Patient Reported Outcomes, Burdens and Experiences (PROBE) Project: development and evaluation of a questionnaire assessing patient reported outcomes in people with haemophilia. Pilot Feasibility Stud. 2018 Feb;4(1):58.

7 von Mackensen S. Quality of life in haemophilia. In: Preedy VR, Watson RR, editors. Handbook of disease burdens and quality of life measures. Heidelberg: Springer; 2010. p. 1895-920.
8 von Mackensen S, Bullinger M; Haemo-QoL Group. Development and testing of an instrument to assess the Quality of Life of Children with Haemophilia in Europe (Haemo-QoL). Haemophilia. 2004 Mar;10(s1 Suppl 1):1725.

9 Young NL, Bradley CS, Blanchette V, Wakefield CD, Barnard D, Wu JK, et al. Development of a health-related quality of life measure for boys with haemophilia: the Canadian Haemophilia Outcomes-Kids Life Assessment Tool (CHO-KLAT). Haemophilia. 2004 Mar;10(s1 Suppl 1):34-43.

10 Neufeld EJ, Recht M, Sabio H, Saxena K, Solem CT, Pickard AS, et al. Effect of acute bleeding on daily quality of life assessments in patients with congenital hemophilia with inhibitors and their families: observations from the dosing observational study in hemophilia. Value Health. 2012 Sep-Oct;15(6):916-25.

11 Wolinsky FD, Wyrwich KW, Nienaber NA, Tierney WM. Generic versus disease-specific health status measures. An example using coronary artery disease and congestive heart failure patients. Eval Health Prof. 1998 Jun; 21(2):216-43

12 Hermans C, Auerswald G, Benson G, Dolan G, Duffy A, Jiménez-Yuste V, et al. Outcome measures for adult and pediatric hemophilia patients with inhibitors. Eur J Haematol. 2017 Aug;99(2):103-11.

13 Bullinger M, Brütt AL, Erhart M, Ravens-Sieberer U; BELLA Study Group. Psychometric properties of the KINDL-R questionnaire: results of the BELLA study. Eur Child Adolesc Psychiatry. 2008 Dec;17(S1 Suppl 1):125-32.

14 Ravens-Sieberer U, Bullinger M. Assessing health-related quality of life in chronically ill children with the German KINDL: first psychometric and content analytical results. Qual Life Res. 1998 Jul;7(5):399-407.
15 Neuner B, Krampe H, McCarthy WJ, Reinke S, Kowalski D, Clausnizer H, et al. Psychometric Properties of a Modified KINDL-R Questionnaire for Adolescents and Adults, and Construction of a Brief Version, the KINDL$\mathrm{A}$ (dult)B(rief) Questionnaire, KINDL-AB Acta Haematol. 2018;140(1):1-9.

$16 \mathrm{Hu} \mathrm{L}$, Bentler PM. Cutoff criteria for fit indexes in covariance structure analysis: conventional criteria versus new alternatives. Struct Equ Modeling. 1999;6(1):1-55.

17 EuroQol Research Foundation. EQ-5D-3L user guide. Available from: https://euroqol org/publications/user-guides.

18 Ellert U, Bellach BM. [The SF-36 in the Federal Health Survey-description of a current normal sample]. Gesundheitswesen. 1999 Dec;61(Spec No):S184-90

19 Ware JE Jr, Sherbourne CD. The MOS 36item short-form health survey (SF-36). I. Conceptual framework and item selection. Med Care. 1992 Jun;30(6):473-83.

20 Evans JD. Straightforward Statistics for the Behavioral Sciences. Belmont, CA, US Thomson Brooks / Cole Publishing Co; 1996.

21 Gnanadesikan R, Kettenring JR. Robust estimates, residuals and outlier detection with multiresponse data. Biometrics. 1972;28(1) 81-124.

22 Kowalski CJ. On the effects of non-normality on the distribution of the sample productmoment correlation coefficient. J R Stat Soc Ser C Appl Stat. 1972;21:1-12.

23 DiStefano C, Motl RW. Further investigating method effects associated with negatively worded items on self-report surveys. Struct Equ Modeling. 2006;13(3):440-64.

24 Motl RW, DiStefano C. Longitudinal invariance of self-esteem and method effects associated with negatively worded items. Struct Equ Modeling. 2002;9(4):562-78. 